\title{
COVID-19 AND HEMATOPOIETIC STEM CELL TRANSPLANTATION: RECOMMENDATIONS FROM THE BRAZILIAN SOCIETY OF BONE MARROW TRANSPLANTATION (SBTMO)
}

\author{
1,2,3 Clarisse M. Machado \\ ${ }^{1}$ Virology Laboratory, Institute of Tropical Medicine, University of São Paulo School of Medicine - ${ }^{2}$ HSCT Program, Amaral \\ Carvalho Foundation - ${ }^{3}$ Instituto Israelita de Ensino e Pesquisa (IIEP), Albert Einstein Hospital
}

Running title: COVID-19: SBTMO recommendations

Correspondence to: Clarisse M. Machado - clarimm@usp.br - Virology Laboratory - Institute Of Tropical Medicine Av. Dr. Enéas de Carvalho Aguiar, 470 - Zip code 05403-000 - São Paulo SP Brazil

\section{INTRODUCTION}

In December 2019, the World Health Organization (WHO) was notified about an outbreak of pneumonia of unknown etiology occurring in the city of Wuhan, China. DNA sequencing of clinical samples from Wuhan patients revealed a new coronavirus showing $82 \%$ sequence homology with severe acute respiratory syndrome coronavirus (SARS CoV) [1]. On January 31st 2020, the WHO declare the situation as a "public health emergency of international interest" due to the rapid expansion of the outbreak in China [2].

On February 12th, the novel coronavirus was named "severe acute respiratory syndrome coronavirus 2 " (SARS- CoV-2) by the International Committee on Taxonomy of Viruses, and "COVID-19" the disease caused by SARS CoV-2 [3]. Initially limited to Wuhan, COVID-19 cases were increasingly reported in several Chinese cities and later in several countries. On February 26th, the first case of COVID-19 was identified in the city of São Paulo, in a 61-year-old patient returning from Italy, one of the countries hard hit by COVID-19 [4]. On March 12, 2020, WHO declared the outbreak as a pandemic [5].

Up to July 2nd, 2020, more than 10.7 million cases of COVID-19 have been confirmed worldwide, with over 515 thousand deaths. Currently, the United States, Brazil and Russia are the countries with the highest number of cases, with the USA being the country with the highest absolute number of deaths (https://coronavirus.jhu.edu/map.html).

Hematopoietic stem cell transplant (HSCT) recipients are beginning to be affected by COVID-19 in Brazil and worldwide. So far, it is not clear whether the disease is similar to that seen in immunocompetent individuals. Likewise, the risk factors for unfavorable outcomes have not been established yet. Data from the EBMT COVID-19 registry suggest less severity in children compared to adults and lethality rates of up to $30 \%$, therefore higher than in the general population (https://www.ebmt.org/covid-19-webinars).

\section{SARS COV-2 INFECTION AND COVID-19}

In the general population, SARS CoV-2 infection may present asymptomatically (up to $30 \%$ of cases) [6], or as a mild, moderate, or severe disease [7]. Symptomatic disease develops in 3 phases: acute or early phase ( 2 to 6 days), progressive phase ( 7 to 19 days) and convalescence (10 to 33 days) [8]. The mean incubation period is 5 days, varying from 1 to 14 days, being around 12 days in $95 \%$ of cases. The most frequent symptoms are fever (89\%), cough (72\%), loss of taste $(71 \%)$ and smell $(68 \%)$, and myalgia (43\%), among others. In general, COVID-19 cases present mild forms of the disease $(80 \%), 15 \%$ may require hospitalization and $5 \%$ will need treatment in an intensive care unit (ICU) [11].

After the first week, patients with COVID-19 may enter the convalescent phase with progressive fading of symptoms, or walk into the progressive phase, with worsening of the clinical picture. Shortness of breath with decreased $\mathrm{O} 2$ saturation and increased respiratory rate are indicators of severe COVID-19 and were observed in $23 \%$ to $35 \%$ of the cases initially reported in China $[7,9]$. Severe disease can quickly evolve to critical conditions requiring mechanical ventilation. In patients who enter the critical phase of the disease lethality rates of $50 \%$ have been observed [12].

Overall lethality rates are around $6 \%$, but exceeded $10 \%$ in some countries such as France (17.9\%), Italy 
(14.4\%), and United Kingdom (14\%) (https://coronavirus.jhu.edu/map.html). In adults requiring hospitalization, the risk factors for COVID-19 mortality are age over 60 years $(O R=1.1 ; p=0.004)$; high SOFA score $(O R=5.7 ; p<0.01)$ and $D$-dimer $>1 \mathrm{mg} / \mathrm{mL}(O R$ 18.4; $p=0.003$ ) (13). According to some authors, SARS CoV-2 viral load can be up to 60 times higher in severe cases compared to mild ones, and could be used as a prognostic biomarker [13)]

Although still uncertain, some preliminary information from early cases of COVID-19 in HSCT recipients suggests that immunocompromised patients may develop a different form of the disease [14].

\section{RESPIRATORY VIRUS AND HSCT}

Although undesirable, respiratory viruses (RV) are well-known seasonal visitors in the HSCT scenario. $\mathrm{RV}$ are a major cause of morbidity and mortality in HSCT recipients and a set of preventive measures is necessary to control transmission.

HSCT staff and patients are familiar with the RV polices in HSCT wards and outpatient units. Among others, information about hand hygiene, cough etiquette, proper use of masks, and self-report of respiratory symptoms are part of continued education programs in most HSCT centers. This fact has represented an advantage over other health services during the COVID-19 pandemic, regarding the compliance with prevention practices.

The present document summarizes the Brazilian Society for Marrow Transplantation (SBTMO) recommendations for management of COVID-19 in the setting of HSCT.

\section{HSCT CENTERS}

In the absence of an effective vaccine, the main prevention strategy is to avoid exposure to SARS CoV-2. HSCT recipients, candidates and donors should limit their exposure to infected individuals as much as possible and strictly follow the prevention practices.

At this point, reinforcement of educative actions is recommended for staff, donors, candidates, relatives and recipients. Continued education staff is recommended to prepare educative materials with written information about COVID-19 pandemic, reminding the measures to control RV transmission, such as hand hygiene, cough etiquette, wearing masks and social distancing.

HSCT centers should have separate areas and teams for COVID-positive and COVID-negative patients, and follow the policies and procedures defined by national authorities, as well as local or institutional policies.

Non-urgent transplants should be postponed, especially for non-malignant diseases. Similarly, non-essential appointments should be canceled or use telemedicine, if possible. Visitors should be prohibited or restricted as much as possible.

HSCT centers should ensure availability of hematopoietic stem cells (SC) by freezing the product before the start of conditioning regimen. If not possible, have an alternative donor as a back-up. Peripheral blood should be preferred as a SC source, unless there is a strong indication for bone marrow.

\section{STAFF}

Health professionals should follow local, institutional and national COVID-19 protocols. The HSCT center should provide personal protective equipment (PPE) and have a trained staff on the management of suspected or confirmed cases of COVID-19.

Staff with respiratory symptoms should be tested for SARS CoV-2. In the event of a diagnosis of COVID-19, the health professional must be away for 14 days, and retested after this period. Resolution of symptoms and two negative PCR tests within a 7-day interval are necessary to return to work. Testing is also recommended in the event of contact with a suspect or proven case of COVID-19. Given the high frequency of asymptomatic infections, periodic testing of staff is recommended to assess the risk of transmission and to better organize the shifts.

Staff should consider wearing uniforms and change street clothes for hospital clothes once arriving in the HSCT unit. Masks are important to limit SARS CoV-2 spread and to reduce the risk for HCW to become infected. Surgical masks are recommended in the hospital, and N95 masks or similar and eye or face shields are mandatory in COVID-19 positive areas [15]. .

Due to the workload in stressful circumstances, the psychological impact of COVID-19 on staff should not be underestimated [16]. The health professional should have regular rest intervals, and receive support from the team regarding stress management to avoid caregiver fatigue. Staff should receive guidance on how to prioritize sleep, maintain good communication with their colleagues, contact with family members, and maintain physical and leisure activities.

\section{DONORS}

Donors within 28 days prior to donation should practice good hygiene, avoid unnecessary travels, 
crowded places and large group meetings. It is recommended that donors are tested for COVID-19 before starting the mobilization procedure.

Donors with COVID-19 must be excluded from donation for 3 months. In case of urgency, earlier collection can be considered if the donor is well, with a negative test and if there is no appropriate alternative donor. Careful risk assessment must be done on a case-by-case basis.

In the event of close contact with a person diagnosed with COVID-19, the donor should be deferred from donation for at least 28 days, and closely monitored for the development of COVID-19 [14].

\section{HSCT CANDIDATES}

Transplant candidates should minimize the risk of SARS CoV-2 infection, ideally through home isolation 14 days prior to conditioning. Avoid unnecessary hospital appointments. Candidates need to be tested for SARS CoV-2 pre-admission, regardless of symptoms. Result must be negative before starting conditioning.

HSCT should be postponed for 3 months in candidates with SARS CoV-2 infection or COVID-19. In case of high-risk disease, the transplant should be postponed 21-28 days and have 2 negative tests with an interval of 24 hours before admission.

In case of contact with a suspect or confirmed case of COVID-19, any procedure (mobilization, collection, conditioning) should be postponed for 14 days (preferably 21), and the candidate monitored for the appearance of symptoms. PCR test must be negative before transplantation.

\section{HSCT RECIPIENTS}

HSCT recipients should avoid exposure to infected individuals, and comply with prevention practices such as hand hygiene and social distancing. If travel is necessary, preference should be given to particular car, avoiding public transportation (metro, bus, train and flights).

All patients, regardless the presence of symptoms, should be tested for SARS CoV-2 before entering HSCT ward. Test for SARS CoV-2 should also be tested in case of contact with a suspected or confirmed case of COVID-19, and whenever respiratory symptoms are present. The PCR test should be repeated if there is a strong suspicion of COVID19 and the test is negative (false negative).

Patients with a positive test for SARS CoV-2 or other respiratory virus should be removed from rooms with laminar flow or rooms with HEPA filter and positive pressure, unless the ventilation can be turned off.

Patients who test positive for SARS Cov-2 in an upper respiratory tract sample should undergo chest $\mathrm{CT}$ and evaluation of oxygenation impairment. Due to the risk of transmission to the healthcare professional, bronchoalveolar lavage (BAL) is not recommended in case of COVID-19, unless co-infection is suspected.

Patients with COVID-19 should comply with contact and droplet precautions, and be kept in isolation for at least 14 days after the disappearance of symptoms. PCR should be repeated weekly to assess duration of shedding and possibility of precautions' clearance.

\section{Diagnosis}

Acute or early phase: The gold standard in the diagnosis of acute SARS CoV-2 infection is RT-PCR. Sensitivity is greatest if respiratory samples are taken between 4 to 10 days from the onset of symptoms. Sampling at the very beginning of the symptoms can lead to false negative results and the test must be repeated after 72 hours in suspected cases. SARS $\mathrm{CoV}-2$ is more frequently detected in bronchoalveolar lavage fluid (93\%). However, due to the high risk of professional exposure, the procedure has not been recommended. Other respiratory samples which show greatest positivity are sputum (72\%), nasal swab (63\%) and saliva (87\%) $[17,18])$

Convalescence phase: RT-PCR may turn negative after the first week of symptoms, and the detection of SARS CoV-2 antibodies can help in the diagnosis of COVID-19. To date, few studies have evaluated the dynamics of specific $\lg \mathrm{M}$ and $\lg \mathrm{G}$ antibodies and the duration of immunity is not well known. IgG antibodies may be detected before the appearance of IgM, or decrease faster than IgM levels [19] , around the eighth week of the disease. Although sensitivity and specificity rates of commercial serological tests around $90 \%$ have been reported [18], it is important to highlight that this is a rapid evolving field and better second-generation tests are awaited.

\section{TREATMENT}

At this point, no clear recommendations can be made about specific therapies in severe cases of COVID-19 due to limited data and unknown risk versus benefit. Also, it is not known whether HSCT recipients with asymptomatic infection or mild cases of COVID-19 can benefit from any specific treatment. 
Immunosuppression and treatment of bacterial, fungal or viral co-pathogens should be maintained.

So far, the management of COVID-19 in HSCT recipients should be based on:

1) Antiviral, preferably within a prospective controlled study. So far, no antiviral drug has shown significant clinical improvement when used in severe cases of COVID-19 [20,21], or has been studied in transplant recipients. Thus, no recommendation can be made at this point. 2) Monitoring of d-dimer for the possible occurrence of endothelial damage, and prophylactic use of heparin (if not contraindicated) to prevent thrombosis. 3) Diagnosis and management of the cytokine storm, by monitoring C-reactive protein, interleukin-6, ferritin, and having established protocols for the introduction of tocilizumab, corticosteroids, immunoglobulins or other drugs for this purpose (https://www.ebmt. org/covid-19-webinars).

Therapy with COVID-19 convalescent plasma has been recently approved in several countries. Convalescent plasma therapy has been shown effective in the influenza H1N1 pandemic, as well as in the 2003 SARS CoV-1 and 2012 MERS CoV epidemics. However, in severe or life threatening cases of COVID-19, a small open-label randomized trial of convalescent plasma did not show improvement in time for clinical response [22]

\section{COVID-19 pandemic evolution}

Leaving China, the pandemic initially reached large urban centers mainly through air transport and major highways. The lack of knowledge about the disease and the high transmissibility of SARS CoV-2, associated with lack of diagnostic tests, occurrence of asymptomatic cases, delay in the implementation of stricter preventive measures, shortage of personal protective equipment, among others, led to an unprecedented increase of COVID-19 cases worldwide.

In Brazil, once local transmission of SARS CoV-2 was established, a migration of COVID-19 cases to the periphery of large cities was observed, and then to cities in the interior of the states, where the num- ber of hospital and ICU beds is smaller. Despite the actions developed to control viral transmission and prevent the overload of the health system, Brazil failed to contain the pandemic and is currently in second place in relation to the absolute number of cases and deaths by COVID-19 (https://coronavirus. jhu.edu/map.html).

Due to the great economic difficulties caused by the pandemic, many states have gradually resumed activities, which invariably leads to localized reappearance of COVID-19 cases.

HSCT centers must prepare for the resumption of activities gradually, always taking into account the local situation. Some items need to be checked before resuming HSCT activities (table1).

\section{COVID 19 and main changes for HSCT centers in Brazil}

We are experiencing a new reality. Many of our routines, staff, physical areas have been modified. Time will tell if forever, or until an effective vaccine or treatment for COVID-19 appears.

In the short term, the main changes for Brazilian HSCT centers are the testing of asymptomatic candidates, donors, patients and companions entering the HSCT unit; ensure increased testing to follow up COVID-19 cases; testing algorithms in case of exposure to suspected or confirmed cases; define protocols for the treatment and management of COVID-19 complications; ensure social distance in outpatient areas; review the need of having patients in support homes; development or improvement of continued education programs on respiratory virus.

Difficult times create opportunities for change. Each center has its peculiarities and should use creativity to adapt these proposals rationally and according to their reality and needs.

These recommendations may be modified at any time based on new information that emerges on the prevention of SARS CoV- 2 and the management of COVID-19. 
Table 1- Check list to resume HSCT center activities during COVID-19 pandemic

\begin{tabular}{c|c}
\hline Decreasing rates of hospitalization and deaths due to COVID-19 in the region for $\geq 14$ days & $\checkmark$ \\
\hline Enough number of ICU and hospital beds in case of an eventual increase of COVID-19 cases & $\checkmark$ \\
\hline Sufficient supply of PPE and diagnostic tests & $\checkmark$ \\
\hline Staff trained to manage COVID-19 cases; & $\checkmark$ \\
\hline Definition of priorities for patients' admission & $\checkmark$ \\
\hline Ensure point of entry screen and social distancing in common areas & $\checkmark$ \\
\hline Revisit hospital visitation policies & $\checkmark$ \\
\hline Definition of telemedicine policies & $\checkmark$ \\
\hline Ensure continued education on RV preventive measures to the staff, patients, candidates and \\
household contacts & $\checkmark$
\end{tabular}

\section{REFERENCES}

1. Ye Z-W, Yuan S, Yuen K-S, Fung S-Y, Chan C-P, Jin D-Y. Zoonotic origins of human coronaviruses. Int J Biol Sci [Internet]. 2020;16(10):1686-97. Available from: https://www.ecdc.europa.eu/sites/default/ files/documents/SARS-CoV-2-risk-assessment-14february-2020.pdf.pdf

2. World Health Organization (WHO). Novel Coronavirus ( 2019-nCoV ) Situation Report Jan 31st [Internet]. 2020. Available from: https://www. who.int/docs/default-source/coronaviruse/situation-reports/20200131-sitrep-11-ncov.pdf?sfvrsn=de7c0f7_4

3. Gorbalenya AE, Baker SC, Baric RS, de Groot RJ, Drosten C, Gulyaeva AA, et al. The species Severe acute respiratory syndrome-related coronavirus: classifying 2019-nCoV and naming it SARS-CoV-2. Nat Microbiol. 2020;5(4):536-44.

4. Centro de Operações de Emergências em Saúde Pública. Coletiva de Imprensa 26 fev 2020 - COVID19 [Internet]. 2020. Available from: https://portalarquivos2.saude.gov.br/images/pdf/2020/fevereiro/26/ COE-COVID19-COLETIVA-DE-IMPRENSA.pdf

5. World Health Organization (WHO). Coronavirus disease 2019 (COVID-19) Situation Report - 52 [Internet]. 2020. Available from: https://www. who.int/docs/default-source/coronaviruse/situation-reports/20200312-sitrep-52-covid-19.pdf?sfvrsn=e2bfc9c0_4
6. Breslin N, Baptiste C, Gyamfi-Bannerman C, Miller R, Martinez R, Bernstein K, et al. COVID-19 infection among asymptomatic and symptomatic pregnant women: Two weeks of confirmed presentations to an affiliated pair of New York City hospitals. Am J Obstet Gynecol MFM. 2020 Apr 9;(January):100118.

7. Qiu H, Wu J, Hong L, Luo Y, Song Q, Chen D. Clinical and epidemiological features of 36 children with coronavirus disease 2019 (COVID-19) in Zhejiang, China: An observational cohort study. Lancet Infect Dis [Internet]. 2020;2019(20):1-8. Available from: http://www.sciencedirect.com/science/article/pii/S1473309920301985

8. Yu F, Yan L, Wang N, Yang S, Wang L, Tang Y, et al. Quantitative Detection and Viral Load Analysis of SARS-CoV-2 in Infected Patients. Clin Infect Dis [Internet]. 2020 Mar 28;1-19. Available from: https://academic.oup.com/cid/advance-article/ doi/10.1093/cid/ciaa345/5812997

9. Huang C, Wang Y, Li X, Ren L, Zhao J, Hu Y, et al. Clinical features of patients infected with 2019 novel coronavirus in Wuhan, China. Lancet. 2020;395(10223):497-506.

10. Yan CH, Faraji F, Prajapati DP, Boone CE, DeConde AS. Association of chemosensory dysfunction and Covid-19 in patients presenting with influenza-like symptoms. Int Forum Allergy Rhinol [Internet]. 2020;1-18. Available from: http://www.ncbi. nlm.nih.gov/pubmed/32279441 
11. Wang $Y$, Wang $Y$, Chen $Y$, Qin $Q$. Unique epidemiological and clinical features of the emerging 2019 novel coronavirus pneumonia (COVID-19) implicate special control measures. J Med Virol. 2020;92(6):568-76.

12. Wu Z, McGoogan J. Characteristics of and important lessons from the coronavirus disease 2019(COVID-19) outbreak in China. Jama. 2020;2019:10.1001/jama.2020.2648.

13. Liu Y, Yan L, Wan L, Xiang T, Le A, Liu J, et al. Viral dynamics in mild and severe cases of COVID-19. Lancet Infect Dis. 2020 Jun;20(6):656-7.

14. Ljungman $P$, Mikulska M, de la Camara R, Basak GW, Chabannon C, Corbacioglu S, et al. The challenge of COVID-19 and hematopoietic cell transplantation; EBMT recommendations for management of hematopoietic cell transplant recipients, their donors, and patients undergoing CAR T-cell therapy. Bone Marrow Transplant [Internet]. 2020; Available from: http://dx.doi.org/10.1038/s41409-020-0919-0

15. Chu DK, Akl EA, Duda S, Solo K, Yaacoub S, Schünemann $\mathrm{HJ}$, et al. Physical distancing, face masks, and eye protection to prevent person-to-person transmission of SARS-CoV-2 and COVID-19: a systematic review and meta-analysis. 2020;6736(20):1-15.

16. Lai J, Ma S, Wang Y, Cai Z, Hu J, Wei N, et al. Factors Associated With Mental Health Outcomes Among Health Care Workers Exposed to Coronavirus Disease 2019. JAMA Netw open. 2020;3(3):e203976.
17. Wang W, Xu Y, Gao R, Lu R, Han K, Wu G, et al. Detection of SARS-CoV-2 in Different Types of Clinical Specimens. JAMA - J Am Med Assoc. 2020;2-3.

18. To KK, Tsang OT-Y, Leung W, Tam AR, Wu T, Lung DC, et al. Temporal profiles of viral load in posterior oropharyngeal saliva samples and serum antibody responses during infection by SARSCoV-2: an observational cohort study. Lancet Infect Dis [Internet]. 2020 Mar;(January). Available from: https://linkinghub.elsevier.com/retrieve/pii/ S1473309920301961

19. Du Z, Zhu F, Guo F, Yang B, Wang T. Detection of antibodies against SARS-CoV-2 in patients with COVID-19. J Med Virol. 2020;57:1-4.

20. Cao B, Wang Y, Wen D, Liu W, Wang J, Fan G, et al. A trial of lopinavir-ritonavir in adults hospitalized with severe covid-19. N Engl J Med. 2020;382(19):1787-99.

21. Wang $Y$, Zhang D, Du G, Du R, Zhao J, Jin $Y$, et al. Remdesivir in adults with severe COVID-19: a randomised, double-blind, placebo-controlled, multicentre trial. Lancet [Internet]. 2020 May 7;395(10236):1569-78. Available from: http:// www.nejm.org/doi/10.1056/NEJMoa2001282

22. Li L, Zhang W, Hu Y, Tong X, Zheng S, Yang J, et al. Effect of Convalescent Plasma Therapy on Time to Clinical Improvement in Patients With Severe and Life-threatening COVID-19. Jama. 2020; 